\title{
Performance characterization of the Caltech compact torus injector
}

\author{
P. K. Loewenhardt, ${ }^{\text {a) }}$ M. R. Brown, ${ }^{\text {b) }}$ J. Yee, and P. M. Bellan \\ Depariment of Applied Physics, California Institute of Technology, Pasadena, California 91125
}

(Received 10 June 1994; accepted for publication 17 October 1994)

\begin{abstract}
A device is described which is designed to refuel tokamaks of major radius $\approx 1 \mathrm{~m}$ and magnetic field $\approx 1 \mathrm{~T}$ by the injection of a compact torus. The injector utilizes an acceleration stage which adds kinetic energy to the compact torus so that it can penetrate the tokamak magnetic field. The performance of the device was found to be optimized in an unexpected parameter regime. It is theorized that in this regime the accelerator takes part in the formation of the CT. A performance threshold with respect to accelerator current will be presented and methods of impurity reduction will be detailed. (C) 1995 American Institute of Physics.
\end{abstract}

\section{INTRODUCTION}

A compact torus (CT), or spheromak, is a toroidal magnetofluid configuration in which plasma is confined by a nearly force-free field structure. This structure, formed by currents flowing in the plasma and in nearby conducting surfaces, consists of a poloidal magnetic field wrapped around a toroidal magnetic field. CTs have recently gained attention in a variety of applications, including fusion reactor refueling and $\mathrm{x}$-ray production. ${ }^{1}$ The minimum magnetic energy state ${ }^{2}$ of a CT provides a unique configuration which has a tremendous strength to weight ratio, allowing it to remain intact even under very high levels of acceleration. For example, typical CTs presently produced in laboratories have withstood up to $10^{11} \times$ the acceleration of the Earth's gravity. ${ }^{1}$ This enables a large amount of kinetic energy to be added to the $\mathrm{CT}$ in a short acceleration stage, allowing large kineticenergy densities to be reached with realistically sized devices.

Reactor refueling requires a CT to reach the core of the tokamak plasma. This means that the CT must have a high enough kinetic-energy density that it can penetrate the magnetic field of the tokamak. This requirement can be stated as $\rho_{\mathrm{CT}} v_{\mathrm{Cr}}^{2} / 2 \geqslant B_{\mathrm{tok}}^{2} / 2 \mu_{0}$ (a pressure balance), where $\rho_{\mathrm{CT}}$ is the CT mass density, $v_{C T}$ the $C T$ velocity, and $B_{\text {tok }}$ the tokamak magnetic-field strength. CTs have previously been injected into the ENCORE tokamak by the Caltech Spheromak Injection experiment to study helicity injection and refueling, ${ }^{3}$ and in the TdeV tokamak by the Compact Toroid Fueller experiment to study refueling. ${ }^{4}$ The former experiment formed the basis of the present Caltech CT injector project, which is designed exclusively for refueling and is in preparation for transport to TEXT (Texas Experimental Tokamak) for refueling experiments. It is a required goal to be able to penetrate a $1 \mathrm{~T}$ tokamak magnetic field before moving the device. Investigations and characterizations presented in this paper therefore took place while the device was free-standing and the CT was allowed to impact on the end of the vacuum vessel. It will be shown that the $B_{\text {tok }}=1 \mathrm{~T}$ goal has been exceeded. In addition, a unique operational regime will be

a) Present address: Applied Materials, M/S 1114, 3320 Scott Blvd., Santa Clara, CA 95054.

b) Present address: Department of Physics and Astronomy, Swarthmore College, Swarthmore, PA 19081. presented where performance of the device is unexpectedly enhanced although the regime was expected to degrade performance.

It is important from a refueling perspective that the injection of a CT introduces enough new fuel but does not disrupt the tokamak plasma. Disruption can occur from injecting an excessive particle inventory, since the injected plasma will be significantly cooler than the strongly heated tokamak plasma. The number of particles in the CT should generally not exceed $\approx 30 \%$ of the tokamak particle inventory. In addition, it is important that high- $Z$ impurities from the electrodes be minimized. These impurities cause radiative cooling of the tokamak plasma and reduce the lifetime of the CT. In the Caltech experiment it was found that the electrode structure could significantly degrade the performance of the device by slowing the CT via the liberation of impurities (addition of mass, impurity line emission, ${ }^{5}$ and resistive damping). It was found that excessive impurities could in fact prevent the CT from reaching the end of the vacuum vessel on unaccelerated shots. Baking the electrodes, radio-frequency discharge cleaning, and running the device with hot electrodes were all used to improve the cleanliness of the electrode structure. After such techniques were implemented, a significant improvement in the velocity and lifetime of the CT were achieved.

\section{COMPACT TORUS FORMATION}

The formation region of the device is shown in Fig. 1. Gas is puffed into the electrode region where it is ionized by high voltage $(8-10 \mathrm{kV})$ applied between the inner and outer electrodes [Fig. 1(b)]. After ionization a large current density $\mathbf{J}$ flows along the inner conductor (producing an azimuthal magnetic field $\mathbf{B}_{\theta}$ ), through the plasma, and into the outer electrode. The plasma is forced from the formation region by a $\mathbf{J} \times \mathbf{B}_{\theta}$ force and exerts pressure upon the applied magnetic field [Fig. 1(c)]. The $J \times \mathbf{B}_{\theta}$ force pushes against the applied magnetic-field ("stuffing flux") tension and the plasma moves forward, carrying the magnetic-field lines with it. The latter effect is caused by the high conductivity of the plasma ("frozen-in-field-line" effects). Magnetic-field lines reconnect behind the plasma pulse [Fig. 1(d)], forming a compact torus (also called spheromak) which is in a state of minimum magnetic energy (nearly all internal forces cancel, with $\mathbf{J} \| \mathbf{B}$ 
a)

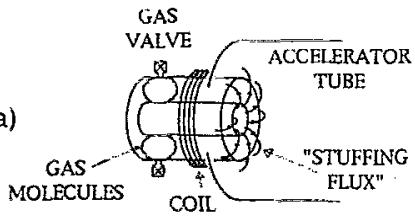

b)

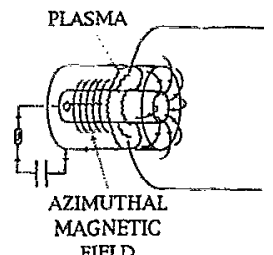

c)

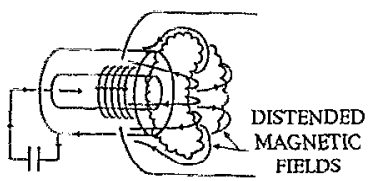

d)

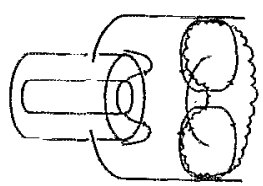

Gas and

Stuffing Flux

Fire Gun Bank

Distend

Stuffing Flux

Formation and

Acceleration

FIG. 1. Formation of a compact torus from a coaxial electrode system: (a) A "stuffing flux" is set up as shown using an electromagnetic coil and a magnetic circuit made of permeable material. Gas is puffed into the interelcctrode volume. (b) A capacitor bank is discharged which ionizes the gas. Current flows down the inner electrode inducing $\mathbf{B}_{\theta}$ and flows through the ionized gas giving a radial current density $\mathrm{J}$. This produces an outward force on the plasma $\mathbf{F}=\mathbf{J} \times \mathbf{B}_{\theta}$. (c) The outward torce pushes the plasma against the stuffing flux, distending the magnetic-field lines. (d) If the outward force is strong enough the magnetic-field lines break and reconnect behind the newly formed compact torus.

internally). The external force preventing radial expansion is provided by image currents in the conductors of the formation and acceleration stages of the device. Formation of a CT is analogous to blowing a soap bubble (see Fig. 1); the stuffing flux magnetic ficld is analogous to the soap film and the plásma pushing against this field is analogous to one's breath.

Since CTs are in an approximately force-free state, internal $\mathbf{J} \times \mathbf{B}$ forces nearly balance, implying that $\mathbf{J} \| \mathbf{B}$ everywhere. The magnetic-field structure is therefore governed by the eigenvalue equation $\nabla \times \mathbf{B}=\lambda \mathbf{B}$ since $\nabla \times \mathbf{B}=\mu_{0} \mathbf{J}$. The quantity $\lambda$ is a constant which is determined from boundary conditions and has the units of inverse length. For conducting boundarics, imagc currents must flow to provide cquilibrium fields. From the above eigenvalue equation one can see that $\lambda=\mu_{0} I / B$ or $\lambda=\mu_{0} I / \Phi$, where $I$ is the formation current and $\Phi$ is the "stuffing" flux. The quantity $\lambda$ is therefore a relation between the formation of a CT and the geometry of the device in which it is formed.

A restricted range of applied magnetic field and plasma current result in the formation of a magnetized plasma "bubble." The formation conditions are characterized by the parameter $\lambda$. It has been found in experiments ${ }^{6}$ that it is necessary to have $\lambda>\lambda_{\text {crit }} \approx \pi / \delta_{\text {gap }}$ ( $\delta_{\text {gap }}$ is the radial distance between inner and outer electrodes) for an isolated CT to be

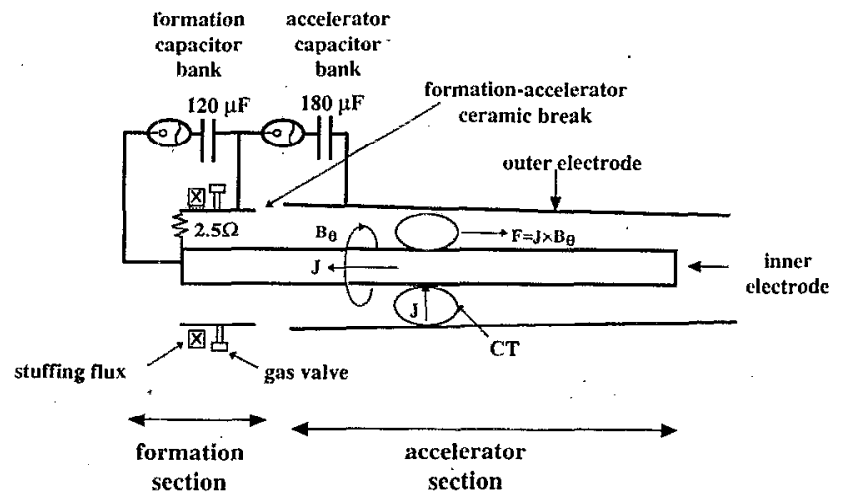

FIG. 2. The basic design of the Caltech CT injector. The break in the outer electrode is an insulating break allowing a second capacitor bank to be fired to accelerate the CT to higher velocities. The compact torus acts like the armature in a coaxial rail gun once in the $2.1 \mathrm{~m}$ Iong accelerator section.

formcd. If experimental parameters are outside of this CT regime, the plasma either cannot overcome the magneticfield tension and only distends the applied magnetic field $\left(\lambda<\lambda_{\text {crit }}\right)$, or the plasma has such a high pressure a CT is not formed ( $\lambda \gg \lambda_{\text {crit }}$, analogous to blowing into a soap film too hard and bursting the film). The latter situation is a Marshall gun $^{7}$ configuration. Once formed, the CT moves out of the formation section at the characteristic Alfven velocity $\left(v_{A} \approx 10-20 \mathrm{~cm} / \mu \mathrm{s}\right)$.

\section{THE CALTECH COMPACT TORUS INJECTOR}

\section{A. Device overview}

A basic schematic of the experimental apparatus is shown in Fig. 2. The formation capacitor bank consists of two $60 \mu \mathrm{F} 20 \mathrm{kV}$ capacitors in parallel (usually operated at $V_{\text {gun }} \approx 8 \mathrm{kV}$, energy $\approx 4 \mathrm{~kJ}$ ). This bank is discharged to ionize the gas puff and drive current to force the CT out of the formation region (it should be noted that the inner electrode in this device is the cathode). After formation a second capacitor bank comprised of three $60 \mu \mathrm{F} 20 \mathrm{kV}$ capacitors in parallel (usually operated at $V_{\text {accel }} \approx 10 \mathrm{kV}$, energy $\approx 9 \mathrm{~kJ}$ ) is fired to accelerate the $\mathrm{CT}$ which then acts like the armature in a coaxial rail gun. The capacitor banks are fired via igni-

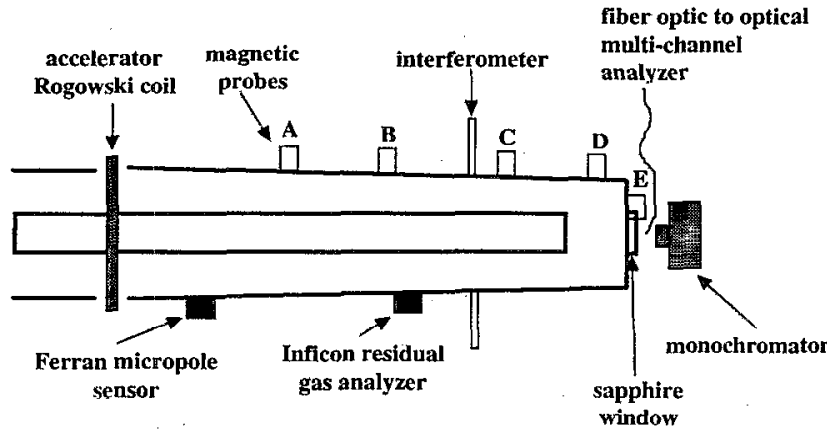

FIG. 3, Schematic of the diagnostics used on the injector. The Leybold Inficon Quadrex 100 residual gas analyzer and the Ferran Scientific micropole sensor are not used during a pulse of the injector but are used afterward to estimate impurity levels in the device. 


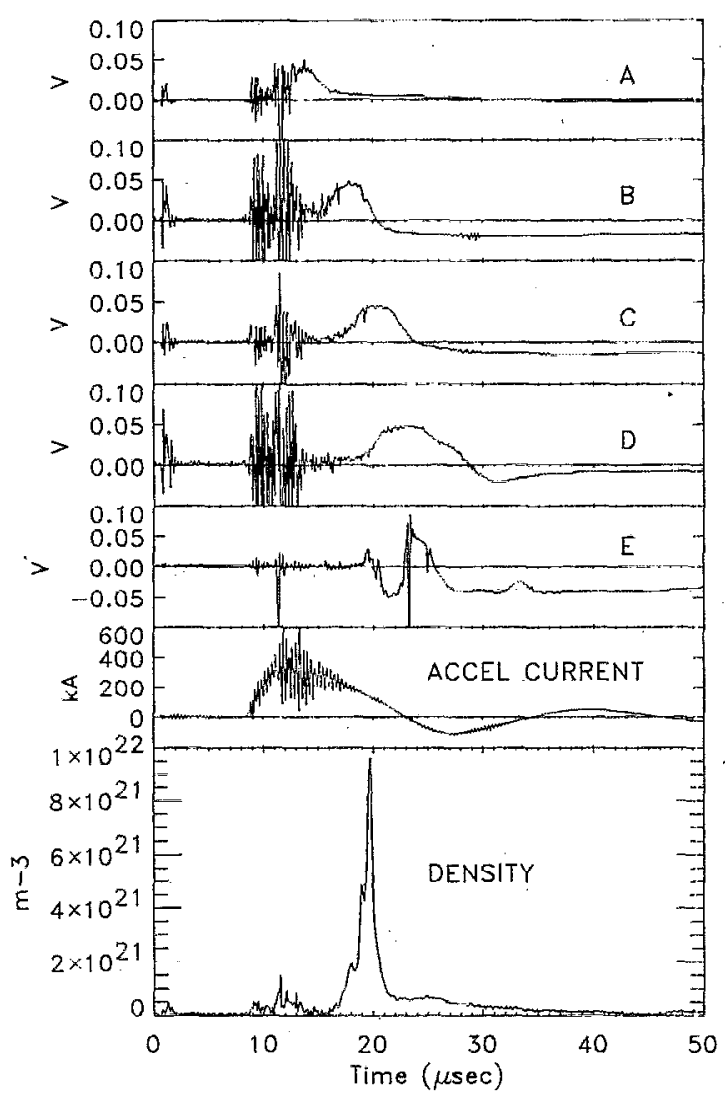

FIG. 4. An example pulse of the Caltech CT experiment. The magnetic signals show the time of flight of the $\mathrm{CT}$, and the interferometer signal shows the line averaged density of the $\mathrm{Cl}$. A Rogowski coil measures the accelerator current. For this example pulse: $V_{\text {accel }}=10 \mathrm{kV}(\approx 9 \mathrm{~kJ})$, $I_{\text {accel,peak }} \approx 350 \mathrm{kA}, V_{\text {gun }}=9 \mathrm{kV}(\approx 5 \mathrm{~kJ}), \Phi=4.6 \mathrm{~m} \mathrm{~Wb}, \lambda \approx 24 \mathrm{~m}^{-1}$. This CT would be able to penetrate a $1.2 \mathrm{~T}$ tokamak magnetic field.

tron switches (National Electronics NL-7703). The triggering circuit uses krytron switches (EG\&G model $\mathrm{KN}-6 \mathrm{~B}$ ) to deliver the trigger pulse to the ignitrons, allowing dependable triggering with $\approx 100-300 \mathrm{~ns}$ rise time. Both capacitor banks are charged by a constant current source (Maxwell CCDS -4 $\mathrm{kJ} / \mathrm{s})$. A low inductance, high-energy capacity voltage snubber $(\approx 2.5 \Omega)$ is connected across the inner and outer electrodes in the formation section. This significantly reduces radiated noise caused by the impedance mismatch between the formation capacitor bank system and the interelectrode space in the short time before gas ionization. Connections from the capacitor banks and the electrodes use low inductance Belden YK-198 cables. Considerable care was taken in the design of the buswork connecting the cables to the capacitor-ignitron arrangement to minimize inductance. This maximized current output and minimized current rise time. A ceramic break isolating the formation from the acceleration sections is placed in the outer electrode.

In the formation section the outer electrode is tungsten spray coated copper while in the accelerator section it is tapered tantalum $(16.8 \mathrm{~cm}$ tapering to $12.7 \mathrm{~cm}$ in diameter over the $\approx 2.1 \mathrm{~m}$ length of the accelerator section) surrounded by a copper tube for-magnetic-flux conservation (this decreases the damping of image currents confining the CT). Tantalum is used because of its low sputtering level.
Electrical isolation between the copper and tantalum in the accelerator section is achieved by a silica mat which allows resistive heating of the tantalum electrode while the more conductive copper provides flux conservation. The outer electrode of the accelerator section is slightly tapered to compress the CT to a higher density state and to enhance its internal magnetic fields. The $6.4 \mathrm{~cm}$ diameter inner electrode consists of a tantalum sheath surrounding a flux conserving copper insert. Physical support of the cantilevered inner electrode is given by a steel tube ( $0.5 \mathrm{~cm}$ wall thickness) which has negligible droop. From the dimensions of the formation section, $\lambda_{\text {crit }} \approx \pi / \delta_{\text {gap }} \approx 60 \mathrm{~m}^{-1}$.

The stuffing magnetic field (typically $\approx 2 \mathrm{kG}$ ) is produced by a magnetic circuit consisting of an iron cage and a ferromagnetic Permandure core inside the inner electrode in the formation section. This magnetic circuit is energized by a coil and produces the correctly configured stuffing flux to form a CT (see Fig. 1). Four fast gas valves ${ }^{8}$ are equally placed azimuthally around the formation stage. These admit an ultrapure ( $<1 \mathrm{ppm}$ impurities) hydrogen gas puff to the formation electrode region. Opening time for the valves is approximately $160 \mu$ s. Hydrogen takes an additional $\approx 120$ $\mu$ s to fill the interelectrode space. Vacuum is provided by a Leybold Turbovac-1000 backed by a Tribodyn-100/38 oilfree pump. Triggering for all the components of the injector is provided by an isolated timing unit via optical fibers.

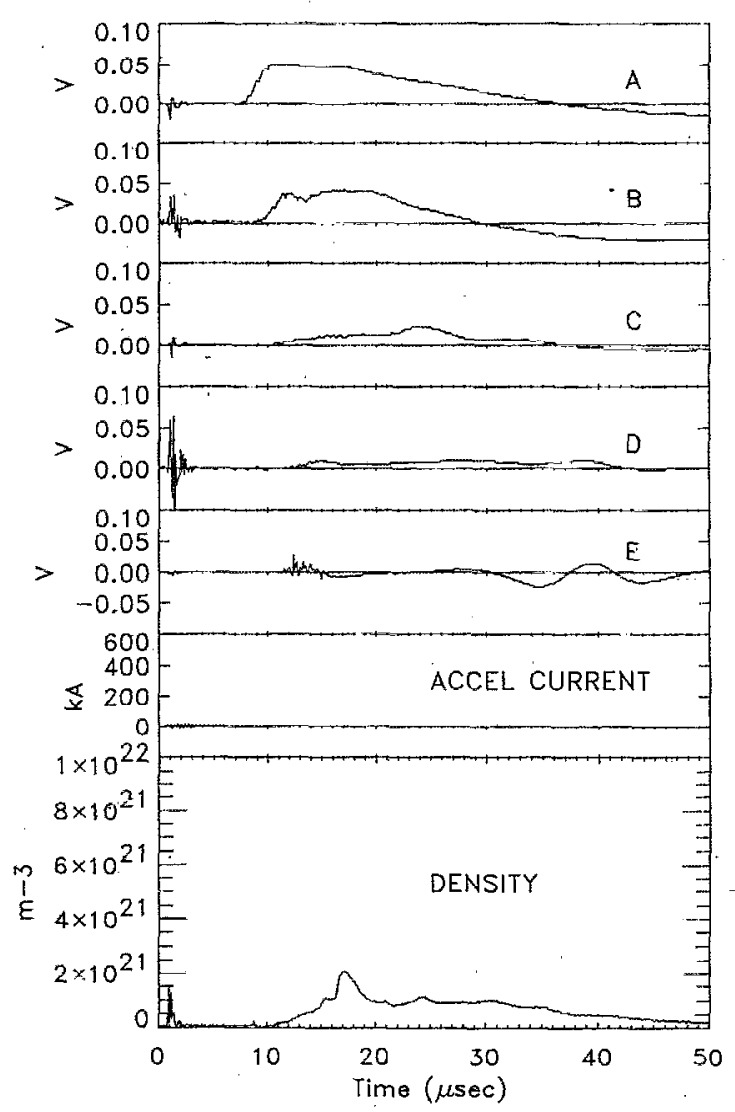

FIG. 5. A pulse of the device with the same parameters as in Fig. 4 but with no accelerator current. Note that the magnetic signal is only significant untiI probe $\mathbf{B}$, implying that an isolated compact torus was not formed. 


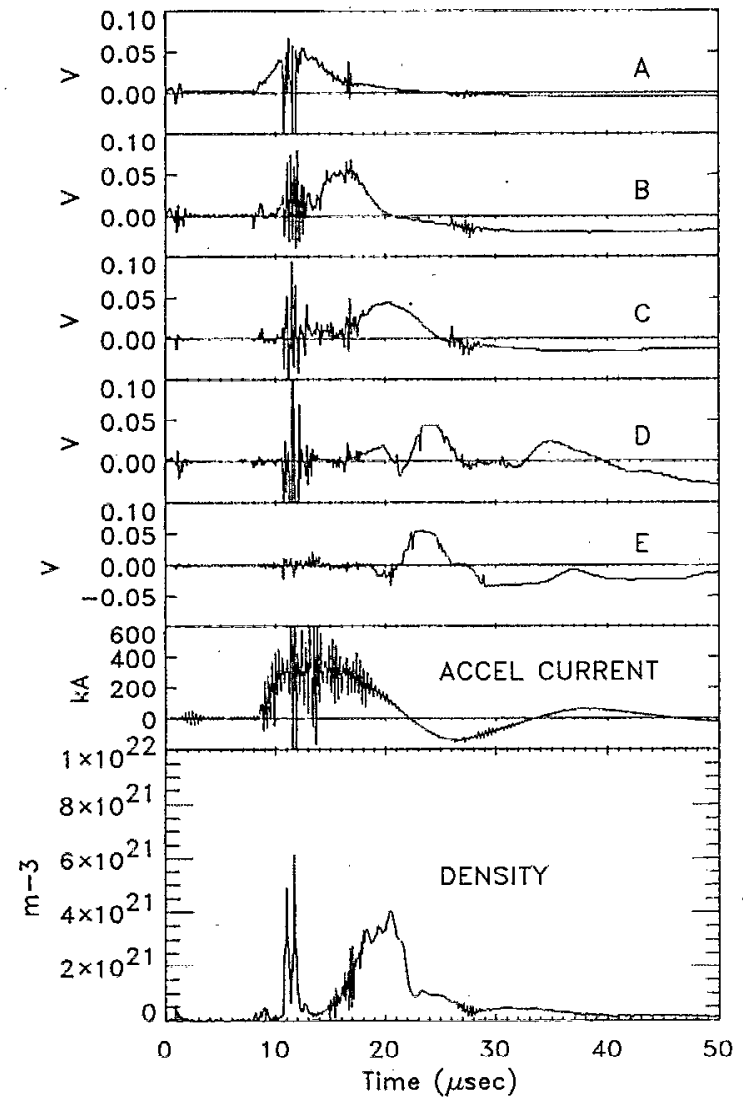

FIG. 6. A pulse of the device with the same parameters as in Fig. 4 but without any heating of the electrodes overnight or helium $\mathrm{CT}$ pulses. This $\mathrm{CT}$ would only be able to penetrate a $0.75 \mathrm{~T}$ tokamak magnetic field.

\section{B. Diagnostics}

Figure 3 is a schematic of the diagnostic systems on the injector experiment. Magnetic probes measuring the axial magnetic field of the passing CT are placed at $0.5 \mathrm{~m}$ increments axially along the acceleration tube. These give an indication of the integrity of the CT and its velocity from timeof-flight measurements. An additional magnetic probe is situated at the end of the vacuum vessel. Line averaged plasma density is measured with a $\mathrm{He}-\mathrm{Ne}$ laser quadrature interferometer located at a port with a radial view of the CT. In the results presented here the density measurements are taken at the port approximately $0.7 \mathrm{~m}$ from the end of the vacuum vessel. A monochromator at the end of the vacuum vessel measures emission line intensities (i.e., $\mathrm{C}_{\mathrm{III}}, \mathrm{O}_{2}, \mathrm{Fe}$ ) through a sapphire window in the endplate of the vacuum vessel. These measurements give a qualitative indication of the level of impurities liberated in the system in each pulse. An optical multichannel analyzer is also used to monitor specific emission lines from the CT. A Rogowski coil is situated at the ceramic break between the formation and accelerator sections to monitor the accelerator current.

\section{Impurity reduction}

A number of other diagnostics are also used to monitor the cleanliness of the device. Ion gauges measure the base pressure and a Leybold Quadrex 100 residual gas analyzer measures the relative amounts of elements in the vacuum

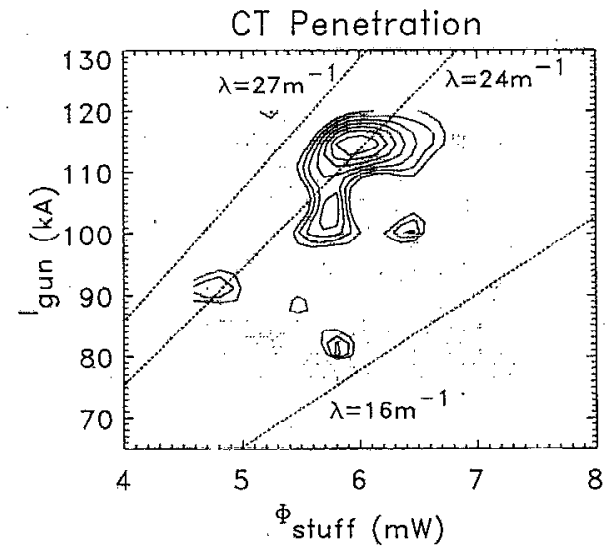

FIG. 7. A contour plot of $B_{\text {tok }}$ as a function of $I_{\text {gun }}$ and $\Phi$. Solid line contours are for $B_{\text {tok }} \geqslant 1.15 \mathrm{~T}$ while dotted line contours are for $B_{\text {tok }}<1.15 \mathrm{~T}$. Lines of constant $\lambda$ are drawn for $\lambda \approx 16,24$, and $27 \mathrm{~m}^{-1}$. Note that performance is optimal for $\lambda \approx 24 \mathrm{~m}^{-1}$. For this scan $V_{\mathrm{accel}}=10 \mathrm{kV}(\approx 9 \mathrm{~kJ})$ $\left(I_{\text {accel,peak }} \approx 350 \mathrm{kA}\right)$.

vessel (see Fig. 3). A Ferran micropole gas analyzer is used to monitor pressures up to $15 \mathrm{mTorr}$; to estimate impurities liberated the exhaust of a CT can be monitored within $10 \mathrm{~s}$ after a pulse.

When the injector is not in use the outer electrode is heated to $\approx 200^{\circ} \mathrm{C}$ by passing approximately $1 \mathrm{kA}(\approx 2 \mathrm{~kW})$ at $60 \mathrm{~Hz}$ through the tantalum shroud. This baking liberates volatile impurities from the electrodes. If a large amount of hydrogen is stored on the electrode surface this gas may be liberated by a passing CT, slowing the $\mathrm{CT}$ and reducing the performance of the injector. To maximize the wall cleanliness the electrodes are baked overnight and then "scoured" by firing approximately 10-20 shots of helium CTs. It has been found that this procedure liberates large amounts of hydrogen trapped in the electrodes, as seen by watching the decrease in the $\mathrm{H}_{\alpha}$ emission line with a Xybion fast shutter camera from pulse to pulse. ${ }^{9}$ Radio-frequency discharge cleaning in argon has also been used on the device, but this procedure was found to deposit tantalum on many internal surfaces, including the endplate sapphire window, and so was

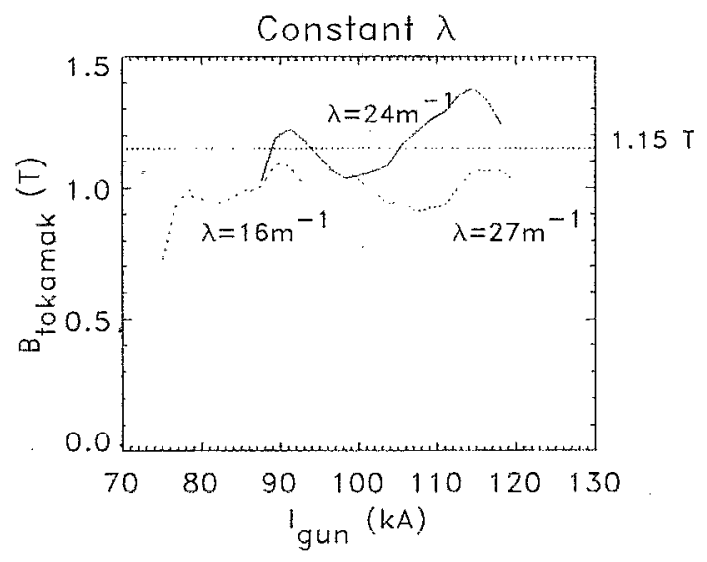

FIG. 8. A plot of $B_{\text {tok }}$ as a function of $I_{\text {gun }}$ for constant $\lambda$. The curves presented are drawn for $\lambda \approx 16,24$, and $27 \mathrm{~m}^{-1}$. Note that although performance varies somewhat for a given $\lambda$, performance is optimal for $\lambda \approx 24$ $\mathrm{m}^{-1}$. For this scan $V_{\text {accel }}=10 \mathrm{kV}(\approx 9 \mathrm{~kJ})\left(I_{\text {accel peak }} \approx 350 \mathrm{kA}\right)$. 


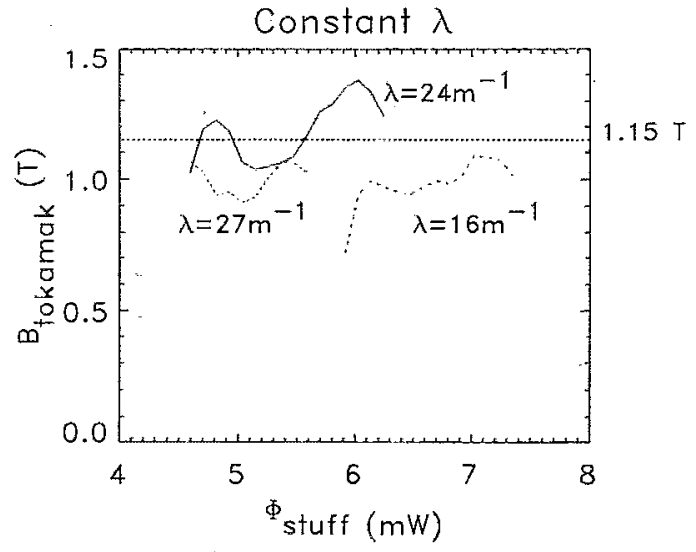

FIG. 9. A plot of $B_{\text {tok }}$ as a function of $\Phi$ for constant $\lambda$. The curves presented are drawn for $\lambda \approx 16,24$, and $27 \mathrm{~m}^{-1}$. Note that although performance varies somewhat for a given $\lambda$, performance is optimal for $\lambda \approx 24 \mathrm{~m}^{-1}$. For this scan $V_{\text {accel }}=10 \mathrm{kV}(\approx 9 \mathrm{~kJ})\left(I_{\text {accel,peak }} \approx 350 \mathrm{kA}\right)$.

abandoned. It was found that performance of the injector was enhanced after carrying out these procedures. The data presented in this paper were all taken after overnight heating and helium CT cleaning.

Care has been taken to eliminate any source of pump oil in the system. When an oil backing pump was used, residual gas analyzer (RGA) measurements showed the cracking pattern characteristic of pump oil. This meant that the oil was reaching the vacuum vessel from the oil based roughing pump via the turbo pump and the gas puff valves (the valves and gas feed system were pumped down by the backing pump at the beginning of each operating session and when switching gas types). An oil-free backing pump (Tribodyn100/38) was installed, which reduced the observed RGA measurements. Performance as presented in this paper was not obtained until after reconfiguration to this oil-free system amongst other improvements. It was also found that features characteristic of improved performance, such as CT lifetime, magnetic signature, and confinement of plasma (as seen by the "sharpness" of the density pulse with respect to time), all improved after attention was paid to impurity reduction. ${ }^{10}$

\section{PERFORMANCE}

Typical diagnostic output is shown in Fig. 4. In this pulse the formation capacitor bank voltage $V_{\text {gun }}=9 \mathrm{kV}(4.9$ $\mathrm{kJ})$ and the accelerator bank voltage $V_{\text {accel }}=10 \mathrm{kV}(9 \mathrm{~kJ})$. Time of flight of the CT can be seen from the axial magneticfield component at positions $\mathrm{A}, \mathrm{B}, \mathrm{C}$, and D each $0.5 \mathrm{~m}$ apart (note that probe $\mathrm{D}$ is beyond the end of the inner electrode). A CT typically takes $2-10 \mu$ s to traverse the distance from the formation section to the end of the vacuum vessel, reaching speeds of $\approx 20-35 \mathrm{~cm} / \mu \mathrm{s}$ near the end of the device. The mass of the accelerated CT ranges from 10 to $200 \mu \mathrm{g}$. The CT in Fig. 4 is comprised of approximately $1.1 \times 10^{20}$ protons corresponding to a mass of $\approx 180 \mu \mathrm{g}$. It is moving at approximately $20 \mathrm{~cm} / \mu \mathrm{s}$ after acceleration (kinetic energy $\approx 3.6 \mathrm{~kJ}$ ), allowing the penetration of $B_{\mathrm{tok}}=1.2 \mathrm{~T}$. This is greater than the $B_{\text {tok }}=1 \mathrm{~T}$ requirement for the device. It is apparent from this example that an isolated $\mathrm{CT}$ is formed and the accelerator section adds a significant amount of kinetic energy to the CT (note the reduction in time of flight between magnetic probe signals as the CT moves further down the accelerator.

The efficiency of the device $\eta$ for the pulse presented in Fig. 4 can be calculated as the ratio of the kinetic energy of the $\mathrm{CT}$ to the energy stored in the formation and accelerator capacitor banks:

$$
=\frac{m_{\mathrm{CT}} v_{\mathrm{CT}}^{2} / 2}{\left(C_{\mathrm{gun}} V_{\mathrm{gun}}^{2}+C_{\mathrm{accel}} V_{\mathrm{accel}}^{2}\right) / 2},
$$

where $m_{\mathrm{CT}}$ is the mass of the CT and $C_{\text {gun }}, C_{\text {accel }}$ are the capacitance of the formation and accelerator sections, respectively. For the shot presented in Fig. 4 the efficiency is approximately $26 \%$.

Performance of the device when initially commissioned was much less ${ }^{11}$ than that presented in Fig. 4. Reaching this performance level required a deviation into an unexpected operational regime for the device. For the data of Fig. 4 the stuffing flux was approximately $4.6 \mathrm{~m} \mathrm{~Wb}$ and $I_{\text {gur }} \approx 90 \mathrm{kA}$, implying that $\lambda \approx 24 \mathrm{~m}^{-1}$ which is considerably less than the critical parameter $\lambda_{\text {crit }} \approx 60 \mathrm{~m}^{-1}$ for this device. Having $\lambda<\lambda_{\text {crit }}$ implies that the plasma cannot completely distend and break off the stuffing magnetic field and therefore a CT cannot be formed. Although the approximation $\lambda_{\text {crit }} \approx \pi / \delta_{\text {gap }}$ may be inaccurate in this situation, Fig. 5, a presentation of an unaccelerated pulse, shows that the formation of the CT is at least partially stuffed. It is believed that the following situation holds in Fig. 4: The plasma distends the stuffing magnetic field out and down into the acceleration section (beyond the formation-accelerator ceramic break) but if unaided cannot completely sever the magnetic field to form an isolated CT. The accelerator capacitor bank is, however, fired near to the time when the plasma and magnetic field are crossing the formation-acceleration ceramic break. This forces current through the plasma adding to the $\mathbf{J} \times \mathbf{B}_{\boldsymbol{\theta}}$ force against the magnetic field. The magnetic tension of the stuffing flux is then completely overcome, allowing the formation of a CT. The improved performance in this regime may arise from the confining magnetic field of the $\mathrm{CT}$ being higher and therefore the $\mathrm{CT}$ is more robust and allows the confinement

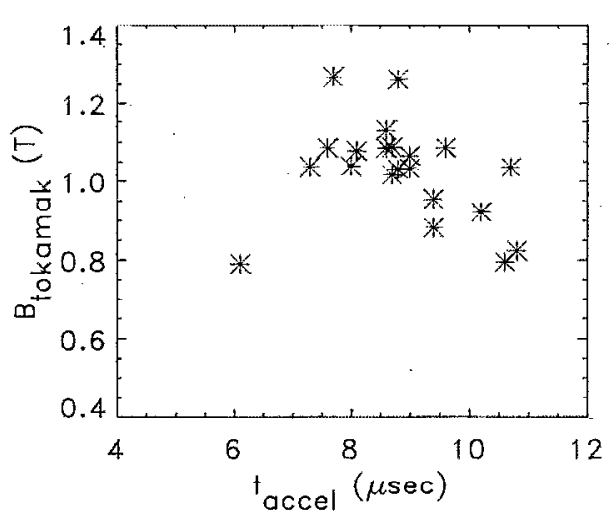

FIG. 10. The variation of $B_{\text {tok }}$ with respect to the timing of the beginning of the accelerator current pulse $t_{\text {accel }}$ for $\lambda \approx 24 \mathrm{~m}^{-1}$. The timing used for the data presented in this paper was in the optimal region. 


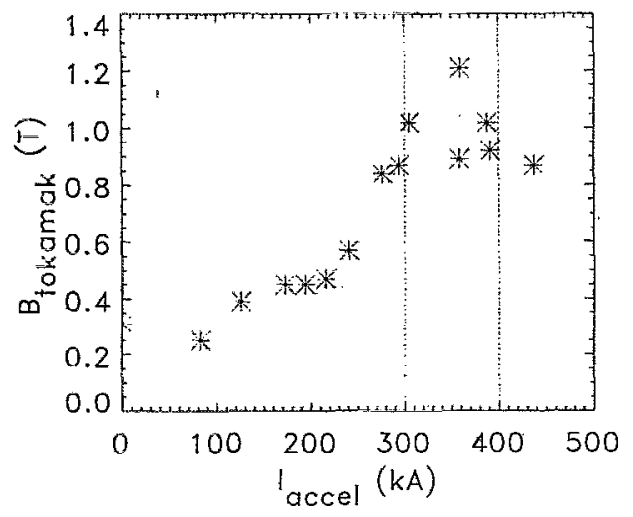

FIG. 11. The variation of $B_{10 k}$ with respect to accelerator current $I_{\text {accel }}$ for $\lambda \approx 24 \mathrm{~m}^{-1}$. Note that there is a threshold above which good performance is reached but below which the injector's behavior is degraded. The dashed vertical lines denote the optimal performance regime.

of higher plasma densities. In addition to this, in the above conceptualization the tension in the magnetic-field lines distended into the accelerator section may add to the $\mathrm{CT}$ acceleration when they are "broken" (i.e., forced reconnection).

An example of the importance of impurity reduction is shown in Fig. 6. The pulse shown was taken with the same operational parameters as Fig. 4 but with no heating overnight or helium CTs fired. Please note that the best CT performance which the device can obtain in this situation only penetrates $B_{\mathrm{tok}}=0.75 \mathrm{~T}$, as opposed to $B_{\mathrm{tok}}=1.2 \mathrm{~T}$ when the electrodes have been treated. Also note the degraded density pulse detected by the interferometer in this regime. A subscquent experiment was carried out where air was bled into the system to raise the internal pressure to $\approx 10^{-3}$ Torr for approximately $5 \mathrm{~min}$ (this introduced impurities such as water). The device was then pumped down to a base level and pulsed. The best level of performance reached in this situation was $B_{\text {tok }}=0.65 \mathrm{~T}$. This degradation of the value of $B_{\text {tok }}$ which the CT can penetrate implies that impurity levels have a significant influence on the injector's performance.

A scan of $I_{\text {gun }}$ and $\Phi$ (a map of $\lambda$ space) was carried out to clarify the regime where the enhanced performance of the device was obtained. Figure 7 shows the result of such a scan for $V_{\text {accel }}=10 \mathrm{kV}(9 \mathrm{~kJ})$. Noting that solid line contours are for $B_{\text {tok }} \geqslant 1.15 \mathrm{~T}$ and dotted contours are for $B_{\text {tok }}<1.15 \mathrm{~T}$, it can be seen that a ridge at constant $\lambda \approx 24 \mathrm{~m}^{-1}<\lambda_{\text {crit }}$ exists for which penetratable $B_{\text {tok }}$ is maximized. Performance far from this ridge and outside of the contour plot was degraded with respect to $\lambda \approx 24 \mathrm{~m}^{-1}$.

Figures 8 and 9 show scans of constant $\lambda$ in which $B_{\text {tok }}$ was determined for varying $\Phi$ and $I_{\text {gun }}$. Data from scans with $\lambda=16,24$, and $27 \mathrm{~m}^{-1}$ (as drawn in Fig. 7) are shown for comparison. It can be seen that there is variation of $B_{\mathrm{tok}}$ with both $\Phi$ and $I_{\text {gun }}$; however, the general trend of $\lambda \approx 24$ $\mathrm{m}^{-1}$ providing maximum $B_{\text {tok }}$ is evident. It was also found that the time at which the accelerator was fired was critical for enhanced performance. Figure 10 is a plot of $B_{\text {tok }}$ as a function of $t_{\text {accel }}$, the time at which the accelerator current rises from zero. Note that $B_{\mathrm{tok}}>1 \mathrm{~T}$ over only a short timing span. Figure 11 is a plot of $B_{\text {tok }}$ as a function of accelerator current. This shows that there is a form of threshold in accelerator current $\left(I_{\text {accel }}>250 \mathrm{kA}\right)$ above which performance is significantly increased. Note that the results presented in this paper are in the realm of higher performance. It has been found that for $I_{\text {accel }}>400 \mathrm{kA}$ performance is somewhat degraded. This is likely caused by a "blow-by" effect where the pushing force from $\mathbf{I}_{\text {accel }} \times \mathbf{B}_{\theta}$ is so great that the CT lifts off the center electrode and the accelerating flux blows by the CT.

\section{ACKNOWLEDGMENTS}

The authors would like to thank Frank Cosso and Larry Begay for their technical assistance. This work was performed under DOE Grant No. DE-FG03-86ER53232.

${ }^{1}$ C. W. Hartman and J. H. Hammer, Phys. Rev. Lett. 48, 929 (1982); T. Jarboe, I. Henins, H. W. Hoida, R. K. Linford, J. Marshall, D. A. Platts, and A. R. Sherwood, ibid. 45, 1264 (1980); J. M. Finn, W. M. Manheimer, and E. Ott, Phys. Fluids 24, 1336 (1981); A. Bondeson, G. Marklin, Z. G. An, H. H. Chen, Y. C. Lee, and C. S. Liu, ibid. 24, 1682 (1981); M. J. Schaffer, ibid. 30, 160 (1987); J. C. Fernández, B. L. Wright, G. J. Marklin, D. A. Platts, and T. R. Jarboe, Phys. Fluids B 1, 1254 (1989); A. Janos, G. W. Hart, and M. Yamada, Phys. Rev. Lett. 55, 2868 (1985); M. N. Rosenbluth and M. N. Bussac, Nucl. Fusion 19, 489 (1979); M. R. Brown and P. M. Bellan, Phys. Rev. Lett. 64, 2144 (1990); M. R. Brown and P. M. Bellan, Phys. Fluids B 2, 1306 (1990); 'C. W. Hartman, J. L. Eddleman, J. H. Hammer, and H. S. McLean, "Experimental Results of Plasma Ring Acceleration and Focusing in the RACE Experiment," Lawrence Livermore National Laboratory Internal Report, 1990 (unpublished).

${ }^{2}$ J. B. Taylor, Rev. Mod. Phys. 58, 741 (1986); C. W. Barnes, J, C. Fernandez, I. Henins, H. W. Hoida, T. R. Jarboe, S. O. Knox, G. J. Marklin, and K. F. McKenna, Phys. Fluids 29, 3415 (1986).

${ }^{3}$ M. R. Brown and P. M. Bellan, Phys. Rev. Lett. 64, 2144 (1990); M. R. Brown and P. M. Bellan, Phys. Fluids B 2, 1306 (1990).

${ }^{4}$ R. Raman, F. Martin, J. Thomas, B. Sawatzky, R. Décoste, J. L. Lachambre, D. Hwang, A. Hirose, C. Williams, and C. Xiao, Bull. Am. Phys. Soc. 38. Paper 6R32 (1993).

${ }^{5}$ C. W. Barnes, I. Henins, H. W. Hoida, T. R. Jarboe, R. K. Linford, J. Marshall, A. R. Sherwood, and M. Tuszewski, "Plasma Impurity Control Studies in CTX," Los Alamos National Laboratory Internal Report, 1991 (unpublishcd).

${ }^{6}$ C. W. Barnes, J. C. Fernandez, I. Henins, H. W. Hoida, T. R. Jarboe, S. O. Knox, G. J. Marklin, and K. F. McKenna, Phys. Fluids 29, 3415 (1986); C. W. Barnes, T. R. Jarboe, G. J. Marklin, S. O. Knox, and I. Henins, Phys. Fluids B 2, 1871 (1990); M. R. Brown, D. M. Cutrer, and P. M. Bellan, ibid. 3, 1198 (1991).

${ }^{7}$ J. Marshall, Phys. Fluids 3, 134 (1960).

${ }^{8}$ J. C. Thomas, D. Q. Hwang, R. D. Horton, J. H. Rogers, and R. Raman, Rev. Sci. Instrum. 64, 1410 (1993).

${ }^{9}$ M. R. Brown and P. M. Bellan, in Plasma Physics and Controlled Nuclear Fusion Research 1992 (Proc. Fourteenth International Conference, Würzburg, 1992), Vol. 3, LAEA, Vienna (1993), p. 475.

${ }^{10}$ M. R. Brown, P. K. Loewenhardt, and P. M. Bellan, Bull. Am. Phys. Soc. 38, Paper 7R25 (1993).

${ }^{11}$ P. K. Loewenhardt, M. R. Brown, and P. M. Bellan, Bull. Am. Phys. Soc. 38, Paper 7R24 (1993). 